\title{
Novel Use of Tracheostomy Shield for Emergency Tracheostomy in Covid 19 Era
}

\author{
Y. K. Kirti ${ }^{1}\left(\right.$ Smita Soni $^{1} \cdot$ J. K. Yashveer $^{1} \cdot$ A. R. Anjali $^{1}$
}

Received: 1 May 2021/Accepted: 10 May 2021/Published online: 17 May 2021

(C) Association of Otolaryngologists of India 2021

\begin{abstract}
To evaluate the problems in doing emergency tracheostomy and tracheostomy care of patients with unknown COVID-19 status. Study the usefulness of the specially designed Tracheostomy Shield. A prospective hospital-based study was conducted at a tertiary care center in India treating COVID and Non COVID patients. The study was done from April 2020 to December 2020. A total of 80 tracheostomy were done using Tracheostomy shield. Open tracheostomies were 38 while 42 patients were already intubated (closed circuit). The Tracheostomy shield was also used in 380 tracheostomy tubes changes. Two patients were found COVID positive on RT-PCR after tracheostomy. There was no scope for testing all tube changes with RT-PCR. The use of our indigenously designed Tracheostomy shield has been effective in reducing the aerosol spread and there was no COVID transmission reported. The use of patient Tracheostomy shield is of uttermost importance in reducing the respiratory droplets splashing on to the surgeon and assistant while doing awake tracheostomy. It is extremely useful while changing tubes as there are chances of false negatives antigen test which might be miss leading. The use of Tracheostomy shield is very important in the current context of new and fast evolving respiratory infections.
\end{abstract}

Keywords Tracheostomy shield .

Tracheostomy in COVID Era - Awake tracheostomy .

Splash of respiratory secretions

Y. K. Kirti

drkirtiyk@gmail.com

1 Department of Otolaryngology, Gandhi Medical College, Bhopal, India

\section{Introduction}

Tracheostomy in COVID-19(Corona virus disease of 2019) times brought out new challenges. Emergency tracheostomy in patients with upper airway obstruction whose COVID status is unknown is a nightmare for the surgeon and anaesthetist. The difficult situations are when patient cannot be intubated or ventilated, with progressive desaturation due to upper airway obstruction. Patients with neck abscess, subglottic foreign bodies, laryngeal malignancies were very well there when the whole medical fraternity was overwhelmed with COVID-19. Medical and surgical resources were also diverted to COVID care. There were lacune in literature as to how to deal with the other lot of patients who could be having laryngeal malignancy with stridor with or without COVID infection. Awake emergency tracheostomy being the only resort to save such patients [1]. Awake tracheostomy in patients with upper air way obstruction does not allow the use of endotracheal intubation, neuromuscular blockers and closed circuits. Changing tracheostomy tubes in outpatient department also poses aerosol generation hazard. Doing urgent tracheostomy in COVID era was a challenge which called for newer methods to deal with the splash and droplets which occurs as the trachea is opened. Changing tubes and doing suction cleaning still remained a grey area. With limited resources, repeated COVID testing was not possible. This problem led to do the present study. 


\section{Methods}

The present study was conducted in a tertiary care centre in central India where both COVID and non-COVID were being treated separately. It was a prospective study from April 2020 to December 2020.

Inclusion criteria- All patients undergoing emergency tracheostomy and patients undergoing tracheostomy tube change in ward and outpatient department whose COVID status was unknown.

The team for open tracheostomy consisted of otorhinolaryngologist, otorhinolaryngology residents, scrub nurse, anaesthetist and anaesthesia residents [2].

The PPEs are to be used based on the risk profile of the health care worker [3]. Rational PPE (personal protective equipment) for tracheostomy (N-95 mask, face shield, fluid resistant coveralls, shoe cover) with proper donning and doffing protocols were followed. For tube change N-95 mask, goggles, gloves and fluid resistant gowns were used. The tube change was performed in the minor OT (operation theatre) attached to the ward and OPD (outpatient department) set up.

A special Tracheostomy shield indigenously designed was used to protect the operating surgeon and the operating room staff form the splash of respiratory secretions in an open tracheostomy. A steel frame was designed to fit over the patient in supine position, the top is covered with glass Fig. 1. The height $-35 \mathrm{~cm}$, breadth- $40 \mathrm{~cm}$ at the head end, breadth- $50 \mathrm{~cm}$ across the chest, length- $50 \mathrm{~cm}$. (tailor made to the existing operating table).

Vertical incision was preferred for a faster bloodless approach. The patient was on spontaneous ventilation with oxygen support. Short acting muscle relaxants were given whenever it was feasible before giving the tracheal incision. A piece of tracheal cartilage was removed. Appropriately sized tracheostomy tube was inserted and cuff inflated. The HME (heat and moisture exchange) filter was

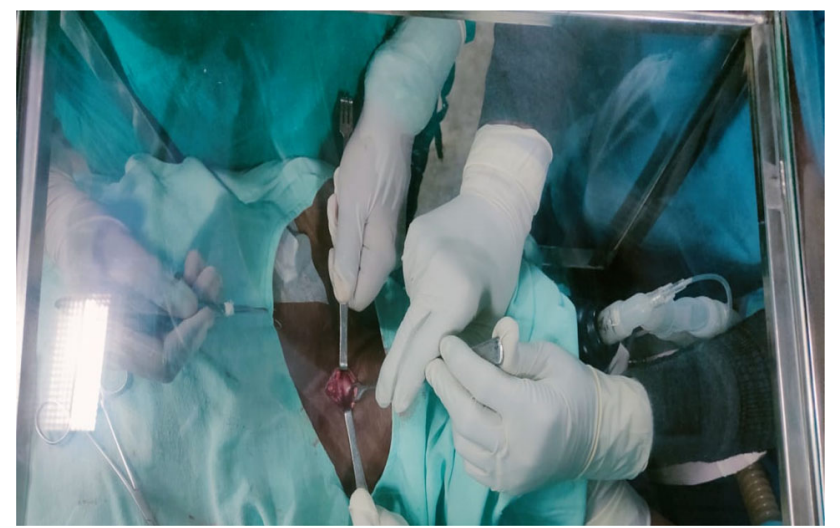

Fig. 1 Clear view and adequate working space below the Tracheostomy shield attached with the circuit and the patient was ventilated, the etco2 was monitored, auscultation was avoided [4].

Diagnosis is usually confirmed by rRT-PCR of combined nasopharyngeal and oropharyngeal swabs. [5].

The whole frame can be sterilized by autoclaving. Since the surgeon looks through the glass his face is protected from unexpected splash without the problem of fogging. The Tracheostomy shield was used in conjunction with regular PPE kits. COVID test was sent but emergency tracheostomy was not delayed and carried out with all COVID protocol.

The Tracheostomy shield was also used for changing tracheostomy tubes in OPD. After suction cleaning, 4\% lignocaine was instilled through the tracheostomy tube to reduce the cough reflex. The new tube is inserted under vision using Tracheostomy shield. The respiratory secretions and droplets get deposited on the under surface of the glass. The same can be wiped and sterilized with sodium hypochlorite solution for next use.

\section{Results}

From April 2020 to December 2020 a total of 80 tracheostomy where done. Open tracheostomies were 38 while 42 patients were already intubated (closed circuit). The Tracheostomy shield was also used in 380 tracheostomy tubes changes.

The advantages of using the Tracheostomy shield were:

1. No direct splash of respiratory secretion on to the surgeon's face shield/visor.

2. Surgeons using spectacles used only goggles as the face shield causes a lot of fogging.

3. The big and small droplets are collected on the glass itself than contaminating the overalls/gown Fig. 2.

4. Gives a sense of security to the OT staff when the patients COVID status is unknown

It was not feasible to send COVID samples in all OPD patients nor to wait for the COVID report to do the emergency tracheostomy/tube changes.

The surgeon, anaesthetist, residents and support staff where well protected, no splashed were experienced, neither was there any fogging issues with adequate working space between the glass and the patient. Two patients were found COVID positive after tracheostomy. There was no scope for testing all tube changes with RT-PCR. No COVID transmission was reported with the use of tracheostomy shield. 


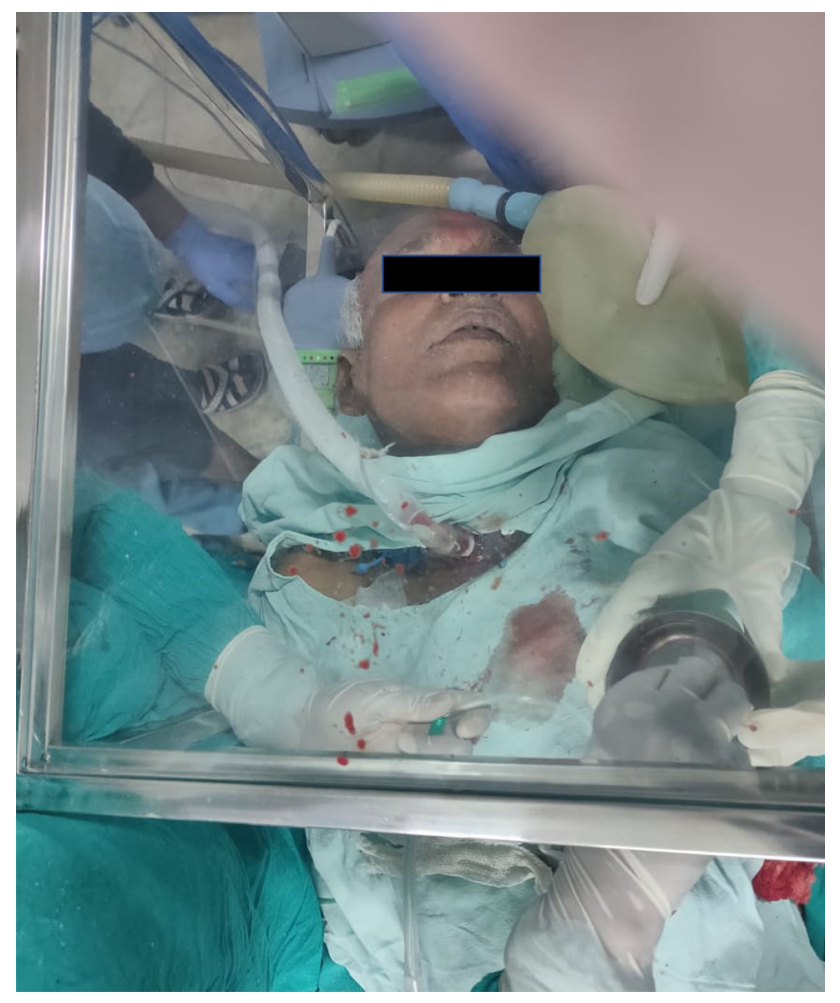

Fig. 2 Blood and secretions get deposited on the glass window of the Tracheostomy shield

\section{Discussion}

Newer strains of old respiratory viruses as well as newer infection which were unheard of are making life of surgeons who deal with airway difficult. A lot has been discussed and published in the recent past regarding the tracheostomy protocols on COVID 19 positive patients.

All the data points to tracheostomy in patients who are on prolonged intubation with a definitive COVID status. This and other questions about when and where to perform these interventions will be influenced by local factors, competencies and experience [6].

The lockdown in India gave rise to new challenges of patients with laryngeal malignancies, neck abscess, subglottic foreign bodies arriving in stridor and desaturation to tertiary care centres. Fear of COVID in the patients mind and in the health care providers resulted in delay in diagnosis, also many hospitals were converted for only COVID care. The other infectious diseases went from bad to worse.

In the initial part of the year 2020 the availability of rapid antigen testing kit was a challenge as well as the time required to get RTPCR reports. Emergency awake tracheostomy without intubation would result in large exposure.
Tracheostomy being an aerosol generating procedure the ideal set up for this procedure would be a dedicated COVID Theatre which operates under negative pressure. The reverse laminar flow is estimated to exchange $90 \%$ of air (removing the generated aerosol) in $6 \mathrm{~min}$ [7]. Of the published cases of tracheostomies done in Singapore, Hong Kong, and Canada during the SARS outbreak, hospitals used enhanced PPE measures in addition to standard PPE, ranging from face shields to powered air-purifying respirators (PAPRs) [2]. Antigen test are not $100 \%$ reliable, RTPCR takes time. In emergency settings the patient has to be treated as a COVID positive unless proved otherwise. There are different issues in the outpatient clinic where the numbers are more and testing is not always possible. The following precautions are mandatory: N95 mask, protective glasses, gown, cap, or hood cap. All disposable equipment that came in contact with the tracheostomy tube or trachea (suction catheters) must be disposed appropriately. The tracheostomy tube should be connected to an HME filter and covered by a surgical mask to minimize risk of aerosol generation and droplet exposure [8,9].

As per COVID protocols closed circuits and muscle relaxants are a must to reduce the aerosolization of the virus. In patients with upper airway obstruction neither sedation nor muscle relaxants can be used, and the patient tends to move a lot. Cumbersome PPE and the fogging make it impossible to operate on a desaturating patient. These challenges combined with the constant threat of COVID made it necessary to use the Tracheostomy shield.

The Tracheostomy shield is a great tool in an otolaryngological setup as it protects against diseases spread through aerosols. The use of face shield/ visor in surgeons who use glasses becomes an issue, due to the temperature difference there is fogging, reducing visibility and obscuring judgement. The patient shield is very helpful in such situations. Having a glass rather than a disposable plastic helps as it gives a clear vision, its stable (does not move like plastic drapes) and ample working space to the surgeon and the assistant. Another disposable plastic drape can be used over the Tracheostomy shield if required to further minimise the aerosol spread, however it becomes cumbersome for the surgeon and suffocating for the patient. The frame is easy to sterilize after each use.

Cessation of respiration using neuromuscular blockers is not possible for tracheostomy tube changes. Hence the shield is very useful in tube changes to reduce the splash of respiratory secretions since it is close to the patient, all the droplets collect on the glass itself. Also repeated testing and tube changes are a costly affair for the patient [10]. The shield helps reduce the aerosolization. When used on a routine basis will definitely help in reducing the spread of not only COVID 19 but also tuberculosis and other 
respiratory infections as otolaryngologist are very susceptible to them.

\section{Conclusion}

The novel use of indigenously designed Tracheostomy shield is protective from splashing of respiratory secretions not only for COVID but for all respiratory diseases. It is a good practise to use the Tracheostomy shield to protect the care givers. PPE is not very convenient and possible in outpatient department where numerous tube changes take place. There is a need to establish new protocols for its use as newer infections will keep challenging the medical fraternity in the future also.

\section{Declarations}

Conflict of interest The authors have no conflict of interest.

Ethical Approval The institutional ethical clearance was obtained for the study.

\section{References}

1. Balakrishnan K, Schechtman S, Hogikyan ND, Teoh AYB, McGrath B, Brenner MJ (2020) COVID-19 Pandemic: What every otolaryngologist-head and neck surgeon needs to know for safe airway management. Otolaryngol Head Neck Surg 162(6):804-808. https://doi.org/10.1177/0194599820919751 (Epub 2020 Apr 14)

2. Tay JK, Khoo ML-C, Loh WS (2020) Surgical considerations for tracheostomy during the COVID-19 pandemic lessons learned from the severe acute respiratory syndrome outbreak. JAMA
Otolaryngol Head Neck Surg. https://doi.org/10.1001/ jamaoto.2020.0764

3. MOHF guidelines for rational use of Personnel protective equipment https://www.mohfw.gov.in/pdf/Additionalguidelineso nrationaluseofPersonalProtectiveEquipmentsettingapproachforHe althfunctionariesworkinginnonCOVIDareas.pdf. Accessed 9 April 2021

4. Heyd CP, Desiato VM, Nguyen SA, O'Rourke AK et al (2020) Tracheostomy protocols during COVID-19 pandemic. Head Neck 42(6):1297-1302

5. Hussain MH, Siddiqui S, Mahmood S, Valsamakis T (2020) Tracheal swab from front of neck airway for SARS-CoV-2; a bronchial foreign body. BMJ Case Rep 13(8):e237787. https://doi.org/10.1136/bcr-2020-237787

6. Jacob T, Walker A, Mantelakis A, Gibbins N, Keane O (2020) A framework for open tracheostomy in COVID-19 patients. Clin Otolaryngol 45(4):649-651. https://doi.org/10.1111/coa.13549

7. Broderick D, Kyzas P, Sanders K, Sawyerr A, Katre C, Vassiliou L (2020) Surgical tracheostomies in Covid-19 patients: important considerations and the "5Ts" of safety. Br J Oral Maxillofac Surg 58(5):P585-589. https://doi.org/10.1016/j.bjoms.2020.04.0081

8. Schultz P, Morvan JB, Fakhry N, Morinière S, Vergez S, Lacroix C, Bartier S, Barry B, Babin E, Couloigner V, Atallah I (2020) French Society of Otorhinolaryngology, Head, Neck Surgery (SFORL) French Society of Head, Neck Carcinology (SFCCF) French consensus regarding precautions during tracheostomy and post-tracheostomy care in the context of COVID-19 pandemic. Eur Ann Otorhinolaryngol Head Neck Dis. 137(3):167-169. https://doi.org/10.1016/j.anorl.2020.04.006

9. Zahran M, Youssef A (2020) Tracheostomy tube change during the COVID-19 pandemic: timing and safety considerations. Egypt J Otolaryngol. https://doi.org/10.1186/s43163-020-00 032-2

10. Chiesa-Estomba CM, Lechien JR, Calvo-Henríquez $\mathrm{C}$ et al (2020) Systematic review of international guidelines for tracheostomy in COVID-19 patients. Oral Oncol 108:104844. https://doi.org/10.1016/j.oraloncology.2020.104844

Publisher's Note Springer Nature remains neutral with regard to jurisdictional claims in published maps and institutional affiliations. 